\title{
Instanton-induced effects in interquark forces, light-front wave functions and formfactors
}

\author{
Edward Shuryak ${ }^{1, *}$ \\ ${ }^{1}$ Center for Nuclear Theory, Stony Brook University, \\ Stony Brook NY 11790 USA
}

\begin{abstract}
Exclusive processes are traditionally described by perturbative hard blocks and "distribution amplitudes" (DAs), matrix elements of operators of various chiral structure and twist. One paper (with I.Zahed) calculate instanton contribution to hard blocks, which is found comparable to perturbative one in few- $\mathrm{GeV}^{2} Q^{2}$ region of interest. Another paper aims at comprehensive wave functions of mesons, baryons and pentaquarks. The last ones are also included as 5-quark component of the baryons. The calculation, using 't Hooft operator, gives $\mathrm{x}$-dependence and magnitude of the antiquark PDF. It explains long standing issue of strong flavor asymmetry of antiquark sea. The third paper (also with I.Zahed)is semi-review on the instanton-sphaleron processes in QCD and electroweak theories, with emphasis on their possible experimental observation via double diffractive events at LHC and RHIC. Insert your english abstract here.
\end{abstract}

\section{Brief history}

The physics of the nonperturbative vacuum of strong interaction started even before QCD. Nambu and Jona-Lasinio (NJL) [1], inspired by BCS theory of superconductivity, have qualitatively explained that strong enough attraction of quarks can break $S U\left(N_{f}\right)_{a}$ chiral symmetry spontaneously and, among many other effects, create near-massless pions.

Instantons, the basis for semiclassical theory of QCD vacuum and hadrons, has been discovered in 1970's [2], and soon t'Hooft has found their fermionic zero modes and formulated his famous effective Lagrangian [3]. Not only it solved the famous " $U_{A}(1)$ problem" - by making the $\eta^{\prime}$ non-Goldstone and heavy - but it also produces a strong attraction in the $\sigma$ and $\pi$ channels. In the framework of the so called instanton liquid model (ILM) [4], it provided a microscopic (QCD-based) basis for chiral symmetry breaking, chiral perturbation theory and the pion properties. Its two parameters

$$
\rho=\frac{1}{3} \mathrm{fm}, \quad n_{I+\bar{I}}=\frac{1}{R^{4}}=1 \mathrm{fm}^{-4}
$$

play the same role as the coupling and the cutoff in the NJL model. These parameters have withstood the scrutiny of time, and describe rather well the chiral dynamics related to pions, the Euclidean correlation functions in the few femtometers range, interacting ensemble of instantons, and much more, see [5] for a review.

\footnotetext{
*e-mail: edward.shuryak@stonybrook.edu
} 
The subject of this talk is several other uses of instantons discussed in recent works. We will consider quark pair production and their role in isospin asymmetry of the nucleon "sea", inclusion of $\bar{I} I$ molecules in mesonic formfactors and forces between quarks in hadrons.

\section{The topological landscape and sphaleron production processes}

There was significant development in understanding the "topological landscape" and relation between instantons and sphaleron production processes, see [6]. Recently interest to it was renewed, due to possible sphaleron production at RHIC/LHC [7]. Let us focus on two main variables, for all static ( 3-dimensional and purely magnetic) configurations of the lowest energy, consistent with the walue of those coordinates. One of the coordinates is the topological Chern-Simons number, and the other is the size squared of the field

$$
N_{C S} \equiv \frac{\epsilon^{\alpha \beta \gamma}}{16 \pi^{2}} \int d^{3} x\left(A_{\alpha}^{a} \partial_{\beta} A_{\gamma}^{a}+\frac{1}{3} \epsilon^{a b c} A_{\alpha}^{a} A_{\beta}^{b} A_{\gamma}^{c}\right), \quad \rho^{2} \equiv \frac{\int d^{3} x \vec{x}^{2} \vec{B}^{2}}{\int d^{3} x \vec{B}^{2}}
$$

If those are kept constant, adding Lagrange multiplies to the action one can find the energy and Chern-Simons number in parametric form

$$
U_{\min }(\kappa, \rho)=\left(1-\kappa^{2}\right)^{2} \frac{3 \pi^{2}}{g^{2} \rho}, \quad N_{C S}(\kappa)=\frac{1}{4} \operatorname{sign}(\kappa)(1-|\kappa|)^{2}(2+|\kappa|)
$$

where $\kappa=0$ corresponds to top of the barrier, known as "sphaleron".

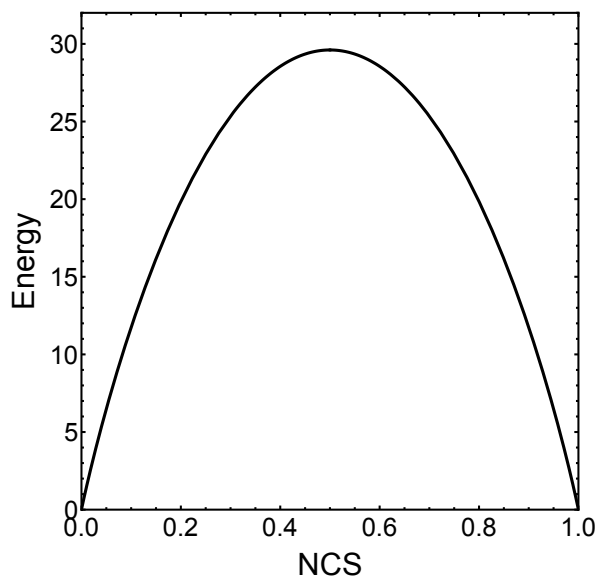

Figure 1. The potential energy $U_{\min }\left(N_{C S}, \rho\right)$ (in units of $1 / g^{2} \rho$ ) versus the Chern-Simons number $N_{C S}$ for the "sphaleron path" between $N_{C S}=0$ and $N_{C S}=1$.

Production of sphaleron-path states can be described semiclassically using instantonantiinstanton "streamline" [8? , 9], and their explosion in Minkowski space-time by [10]. The new discussion is about estimates of how one can produce QCD sphalerons as topologicallycharged clusters in double-diffractive events, with a cluster of few $\mathrm{GeV}$ mass at the center. Its decay modes into 3 mesons were calculated using t'Hooft Lagrangian. 


\section{Mesons and baryon light-front wave functions and isospin asymmetry of the nucleon "sea"}

High energy processes, like famous electron-nucleon deep inelastic scattering, produced rich "parton phenomenology", in form of parton distribution functions (PDFs), transverse distribution functions (TDFs) etc. Those are certain matrix elements of light front wave functions (LFWFs), which, however, did not obtained much attention. Furthermore, understanding of light front Hamiltonians is only in its initial stage.

In [11] I have calculated LFWFs for several mesons and baryons, including 5-quark component of the latter related to the isospin asymmetry puzzle. Canonical process of quark pair production via gluons is "flavor blind", and yet the antiquark sea of the nucleon is very asymmetric, as shown in Fig.2 (right). As noticed in [12], the t'Hooft Lagrangian is strongly flavor asymmetric, say $d$ quark can produce $\bar{u} u$ pair (Fig.2 left) but not $\bar{d} d$. If it would be the only process, the $\bar{d} / \bar{u}$ ratio would be 2 , as there are two $u$ quarks and only one $d$ in the nucleon: not far from the data at certain $x$.

Calculating admixture of 5-q states to the nucleon LFWF, I was able to quantify this effect and get the magnitude of the asymmetry and shape of first-generation antiquarks in agreement with the data.
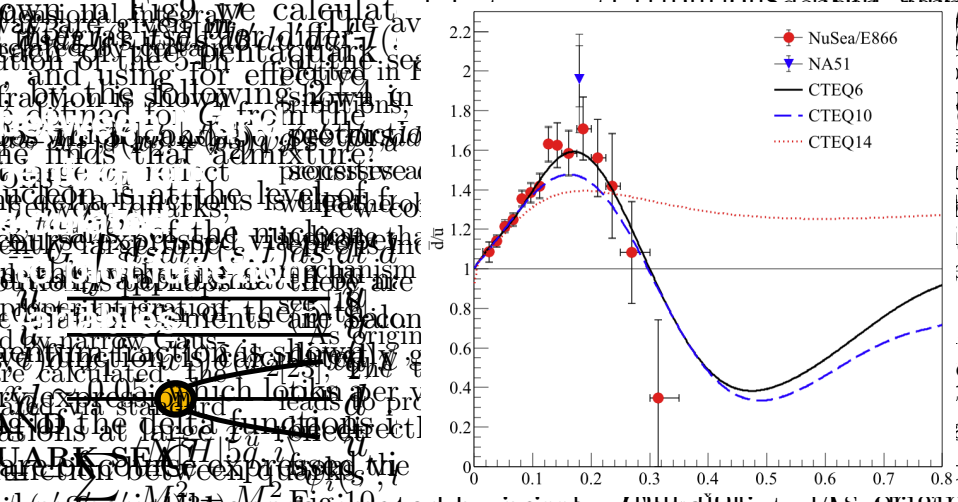

Figure 2. Sea generation via t'Hooft Lagrangian (left) and the data on the $\bar{d} / \bar{u}$ ratio versus $x$, right.

\section{4 "Dense instanton ensemble"}

The original instanton liquid model focused on quark condensate and therefore relatively stand-alone instantons, whose zero modes gets collectivized. But instanton ensemble also includes close instanton-antiinstanton pairs, as it is also observed on the lattice. The stand-alone instantons are seen via "deep cooling", during which the instanton-antiinstanton molecules get annihilated. In so far, the application of the "molecular component" of the semiclassical ensemble was made only in connection to phase transitions in hot/dense matter. Indeed, this component is the only one which survives at temperatures $T>T_{c}$, where chiral symmetry is restored. Account for both components together started with [13]. The "molecular component" was also shown to be important at high baryonic densities, where it contributes to quark pairing and color superconductivity [14]. Close $\bar{I} I$ pairs has been qualitatively studied in recent work in [15] which studied their evolution during cooling, see Fig.3. Extrapolated to zero cooling (left side of the plots) one sees that while the instanton size fits previous expectations (1), the density seems to actually be significantly larger. 

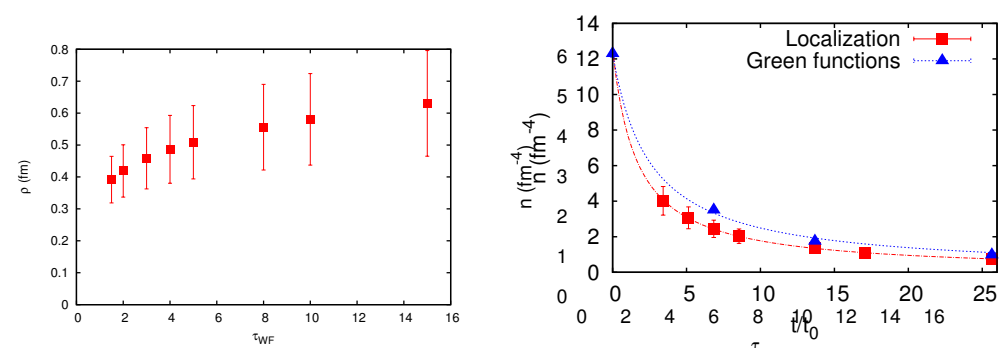

Figure 3. The dependence of the mean instanton sizes (upper plot) and density (lower plot) on the gradient flow cooling time $\tau$ (arbitrary units). The quantum vacuum of course corresponds to an extrapolation to $\tau \rightarrow 0$.
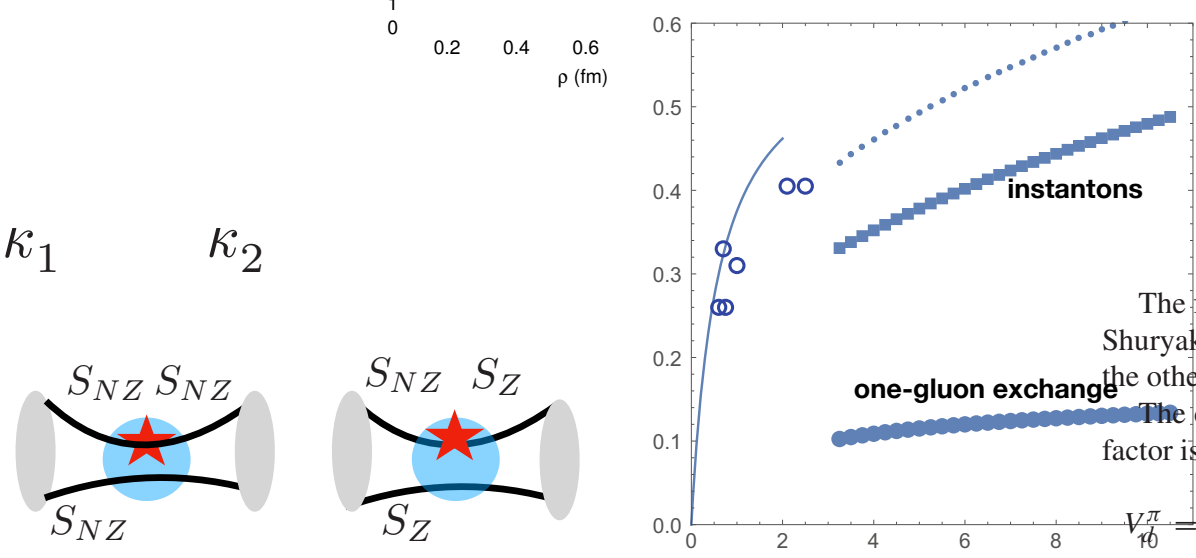

Figure 4. Left: two diagrams with quark propagators in the instanton fields. Right: vector formfactor of the pion $Q^{2} F_{\pi}\left(Q^{2}\right)$. Open points correspond to some experimental data points, thin curve on the left is standard dipole fit. The dotted line on the top is the sum of perturbative and instanton contributions

\section{Formfactors: including instantons in hard block}

More recently, we have explored non-perturbative contributions to the "hard block" of mesonic form factors [16]. We calculated photon, scalar, graviton and dilaton FFs for pion, rho and scalar $a_{0}$ (brother of $\eta$ ?). The field at the instanton center is rather strong, with $\rho=1 / 3 \mathrm{fm}$ is large

$$
G_{r m s} \equiv \sqrt{\left(G_{\mu \nu}^{a}(0)\right)^{2}}=\sqrt{192} / \rho^{2} \approx 5 \mathrm{GeV}^{2},
$$

comparable to $Q^{2}$ is the so called semi-hard region studied.

One example, which was studied a lot experimentally, is electromagnetic pion formfactor, shown in Fig.4 right. It was found that they are mostly dominated by the diagram with three propagator made of non-zero Dirac modes (the left one on the left), not the one with Dirac zero modes. Therefore we included contributions of "dense instanton liquid", taking the diluteness parameter $\kappa=\pi^{2} n_{I+\bar{I}} \rho^{4}$ to one, in plots like Fig. 4 right. As one can see, in this version the sum of perturbative and instanton-induced formfactor reproduce the data. 
The expressions for perturbative, non-zero-mode and zero mode parts are

$$
\begin{aligned}
& V_{a}^{\pi}\left(Q^{2}\right)=\epsilon_{\mu}(q)\left(p^{\mu}+p^{\prime \mu}\right)\left(e_{u}+e_{\bar{d}}\right)\left[\left(\frac{2 C_{F} \pi \alpha_{s} f_{\pi}^{2}}{N_{c} Q^{2}}\right)\right. \\
& \times \int d x_{1} d x_{2}\left(\frac{1}{\bar{x}_{1} \bar{x}_{2}+m_{\text {gluon }}^{2} / Q^{2}}\right)\left(\varphi_{\pi}\left(x_{1}\right) \varphi_{\pi}\left(x_{2}\right)\right. \\
& \left.\left.+2 \frac{\chi_{\pi}^{2}}{Q^{2}}\left(\varphi_{\pi}^{P}\left(x_{1}\right) \varphi_{\pi}^{P}\left(x_{2}\right)\left(\frac{1}{\bar{x}_{2}+E_{\perp}^{2} / Q^{2}}-1\right)+\frac{1}{6} \varphi_{\pi}^{P}\left(x_{1}\right) \varphi_{\pi}^{\prime T}\left(x_{2}\right)\left(\frac{1}{\bar{x}_{2}+E_{\perp}^{2} / Q^{2}}+1\right)\right)\right)\right] \\
& V_{c}^{\pi}=\quad \epsilon_{\mu}(q)\left(p^{\mu}+p^{\prime \mu}\right)\left(e_{u}+e_{\bar{d}}\right)\left[\frac{\kappa \pi^{2} f_{\pi}^{2} \chi_{\pi}^{2}}{N_{c} M_{Q}^{2}}\left\langle\rho^{2} \mathbb{G}_{V}(Q \rho)\right\rangle\right. \\
& \left.\quad \times \int d x_{1} d x_{2} \bar{x}_{1}\left(\varphi_{\pi}^{P}\left(x_{1}\right) \varphi_{\pi}^{P}\left(x_{2}\right)-\frac{1}{36} \varphi_{\pi}^{\prime T}\left(x_{1}\right) \varphi_{\pi}^{\prime T}\left(x_{2}\right)\right)\right] \\
& \quad V_{d}^{\pi}=-\epsilon_{\mu}(q)\left(p^{\mu}+p^{\prime \mu}\right)\left(e_{u}+e_{\bar{d}}\right) \\
& \times\left[\left(\frac{1}{N_{c}^{2}\left(N_{c}+1\right)}\right) \frac{4 \kappa \pi^{2} f_{\pi}^{2} \chi_{\pi}^{2}}{3 M_{Q}^{2}}\left\langle\rho^{2} \frac{K_{1}(Q \rho)}{Q \rho}\right) \int d x_{1} d x_{2} \varphi_{\pi}^{P}\left(x_{1}\right) \varphi_{\pi}^{\prime T}\left(x_{2}\right)\right]
\end{aligned}
$$

Next-twist distribution amplitudes $\varphi_{\pi}^{P}, \varphi_{\pi}^{T}$ are associated with pseudoscalar $\gamma_{5}$ and tensor $\sigma^{\mu \nu}$ ones, the leading twist $\varphi_{\pi}$ is standard matrix element of the axial current $\gamma_{\mu} \gamma_{5}$. For the plot all three are taken to be just constant, independent on $x$. (Therefore all contributions of the tensor DA, appearing as a derivative over $x$, becomes actually vanish.)

\section{Instanton-induced inter-quark forces}

Historically, hadronic spectroscopy got to solid foundation in 1970's, with discoveries of nonrelativistic quarkonia made of heavy $c, b$ quarks. In the first approximation, those are well described by simple Cornell potential

$$
V_{\text {Cornell }}(r)=-\frac{4 \alpha_{s}}{3} \frac{1}{r}+\sigma_{T} r
$$

which correctly attributes the short-distance potential to perturbative gluon exchange, and its large distance $O(r)$ contribution to the tension of a confining flux tube (the QCD string). One of the issues to be discussed in this paper is the nonperturbative origin of the inter-quark interaction at intermediate distances $r \sim 0.2-0.5 \mathrm{fm}$.

Later developments $[17,18]$ connected interquark potential to correlator of Wilson lines (central)

$$
e^{-V_{C}(r) T}=\left\langle W\left(\vec{x}_{1}\right) W^{\dagger}\left(\vec{x}_{2}\right)\right\rangle
$$

Spin-dependent forces were related to such correlators with two magnetic fields $\left(V_{S S}, V_{\text {tensor }}\right)$, or magnetic and electric fields for spin-orbit one. To evaluate such nonlocal quantities one needs to use lattice simulation, or rely on certain model of the vacuum fields. In Fig.5 (left) we show "dense instanton model" to the central potential, compared to linear potential and its version from [19] including string quantum vibrations (resummed "Lusher terms"). One can see that instantons can comlpement the flux tube at intermediate distances.

One problem with electric flux tube model is that it does not provide magnetic fields, while instantons are self-dual and have $B$ needed for spin forces. We calculated the instanton contributions to spin forces, see $V_{S} S$ comparison of lattice potential, perturbative and 

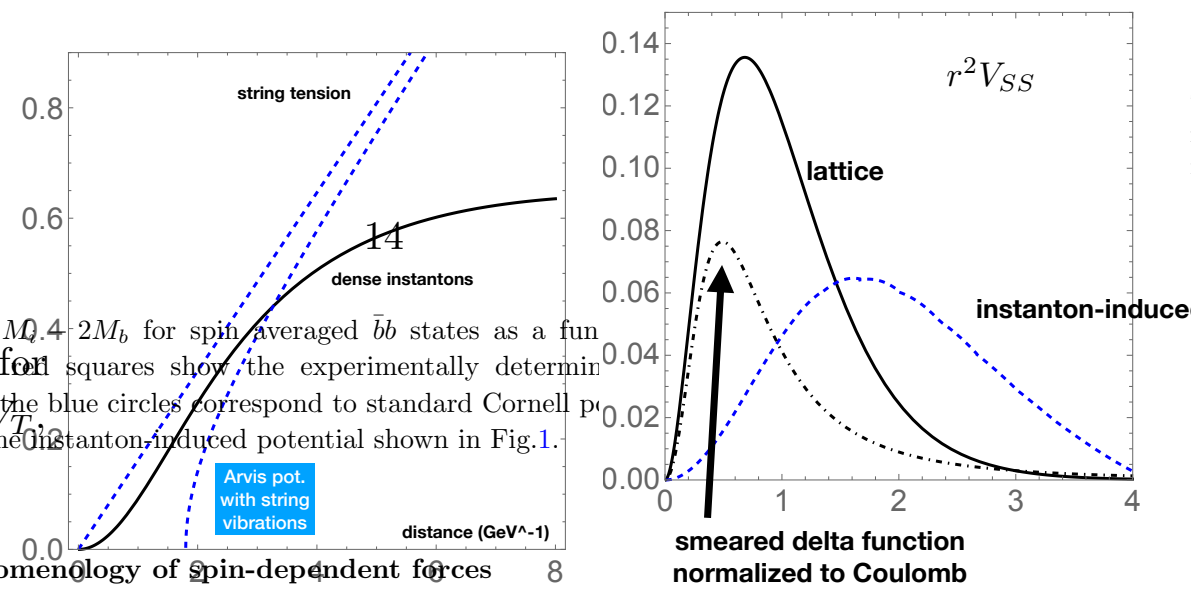

\begin{tabular}{|c|c|c|c|c|c|}
\hline flavors & $M_{\Upsilon}-M_{\eta_{b}}$ & $M_{J / \psi}-M_{\eta_{c}}$ & $M\left(D^{*}\right)-M(D)$ & $M\left(K^{*}\right)-M(K)$ & $M(\rho)-M(\pi)$ \\
\hline $\operatorname{Exp}$ & 61. & 116. & 137. & 398. & 636. \\
$\left\langle V_{S S}^{\text {lat }} / 3 M_{1} M_{2}\right\rangle$ & 46. & 108. & 98. & 170. & \\
$\left\langle\vec{\nabla}^{2} V_{C} / 3 M_{1} M_{2}\right\rangle$ & 28. & 58. & 48. & 82. & \\
$\left\langle\vec{\nabla}^{2} V_{\text {inst }} / 3 M_{1} M_{2}\right\rangle$ & 7. & 30. & 48. & 90. & \\
\hline
\end{tabular}

Figure 5. The central potential $V_{c}$ (left) and spin-spin $r^{2} V_{S S}(r)$ (right) versus distance $r,\left(\mathrm{GeV}^{-1}\right)$. The lattice result is one of the parameterization given in [20], the perturbative is Laplacian of (regulated) Coulomb term.

instanton-induced in Fig.5 (right). Note that the area below the perturbative and instantoninduced terms is comparable. The corresponding matrix elements (using Cornell wave functions with proper quark masses) is shown in the table. One can see that e.g. for charmonium the magntitude of spin-spin term is in agreement with lattice and the level splitting. We also considered $L=1$ families of mesons, from heavy to light, and considered other spindependent potentials. We also discussed $\bar{I} I$ molecules, which provide somewhat different potentials due to different pictures of their fields.

However for heavy-light and light-light cases this $V_{S S}$ is not enough: the missing part is then attributed to part of the quark propagators containing zero modes ( $t$ 'Hooft Lagrangian). It works well for heavy-light and the pions, as expected.

We undertake this study of inter-quark forces in [21], preparing to calculate the light-front Hamiltonian and corresponding light-front wave functions [22].

\section{References}

[1] Y. Nambu and G. Jona-Lasinio, Phys. Rev. 122 (1961), 345-358 doi:10.1103/PhysRev.122.345

[2] A. A. Belavin, A. M. Polyakov, A. S. Schwartz and Y. S. Tyupkin, Phys. Lett. B 59 (1975), 85-87 doi:10.1016/0370-2693(75)90163-X

[3] G. 't Hooft, Phys. Rev. D 14 (1976), 3432-3450 [erratum: Phys. Rev. D 18 (1978), 2199] doi:10.1103/PhysRevD.14.3432

[4] E. V. Shuryak, Nucl. Phys. B 203 (1982), 140-156 doi:10.1016/0550-3213(82)90480-1 
[5] T. Schäfer and E. V. Shuryak, Rev. Mod. Phys. 70 (1998), 323-426 doi:10.1103/RevModPhys.70.323 [arXiv:hep-ph/9610451 [hep-ph]].

[6] D. M. Ostrovsky, G. W. Carter and E. V. Shuryak, Phys. Rev. D 66 (2002), 036004 doi:10.1103/PhysRevD.66.036004 [arXiv:hep-ph/0204224 [hep-ph]].

[7] E. Shuryak and I. Zahed, [arXiv:2102.00256 [hep-ph]].

[8] J. J. M. Verbaarschot, Nucl. Phys. B 362 (1991), 33-53 [erratum: Nucl. Phys. B 386 (1992), 236-236] doi:10.1016/0550-3213(91)90554-B

[9] V. V. Khoze and A. Ringwald, CERN-TH-6082-91.

[10] E. Shuryak and I. Zahed, Phys. Rev. D 67 (2003), 014006 doi:10.1103/PhysRevD.67.014006 [arXiv:hep-ph/0206022 [hep-ph]].

[11] E. Shuryak, Phys. Rev. D $\mathbf{1 0 0}$ (2019) no.11, 114018 doi:10.1103/PhysRevD.100.114018 [arXiv:1908.10270 [hep-ph]].

[12] A. E. Dorokhov and N. I. Kochelev, Phys. Lett. B 304 (1993), 167-175 doi:10.1016/0370-2693(93)91417-L

[13] E. M. Ilgenfritz and E. V. Shuryak, Nucl. Phys. B 319 (1989), 511-520 doi:10.1016/0550-3213(89)90617-2

[14] R. Rapp, T. Schäfer, E. V. Shuryak and M. Velkovsky, Phys. Rev. Lett. 81 (1998), 53-56 doi:10.1103/PhysRevLett.81.53 [arXiv:hep-ph/9711396 [hep-ph]].

[15] A. Athenodorou, P. Boucaud, F. De Soto, J. Rodríguez-Quintero and S. Zafeiropoulos, JHEP 02 (2018), 140 doi:10.1007/JHEP02(2018)140 [arXiv:1801.10155 [hep-lat]].

[16] E. Shuryak and I. Zahed, Phys. Rev. D 103 (2021) no.5, 054028 doi:10.1103/PhysRevD.103.054028 [arXiv:2008.06169 [hep-ph]].

[17] C. G. Callan, Jr., R. F. Dashen, D. J. Gross, F. Wilczek and A. Zee, Phys. Rev. D 18 (1978), 4684 doi:10.1103/PhysRevD.18.4684

[18] E. Eichten and F. Feinberg, Phys. Rev. D 23 (1981), 2724 doi:10.1103/PhysRevD.23.2724

[19] J. F. Arvis, Phys. Lett. B 127 (1983), 106-108 doi:10.1016/0370-2693(83)91640-4

[20] T. Kawanai and S. Sasaki, Phys. Rev. D 92 (2015) no.9, 094503 doi:10.1103/PhysRevD.92.094503 [arXiv:1508.02178 [hep-lat]].

[21] E. Shuryak and I. Zahed, [arXiv:2110.15927 [hep-ph]].

[22] E. Shuryak and I. Zahed, [arXiv:2111.01775 [hep-ph]]. 